\title{
Kinematic Analysis of 6 DOF Articulated Robotic Arm
}

\author{
T. Aravinthkumar ${ }^{1, *}$, M. Suresh ${ }^{1}$, B. Vinod ${ }^{1}$ \\ ${ }^{1}$ Department of Robotics and Automation Engineering, PSG College of Technology, Coimbatore-641004, India \\ *Corresponding author email: aravinthkumar.thangavelu@gmail.com \\ DOI: https://doi.org/10.34256/irjmt2111 \\ Received: 02-01-2021, Revised: 15-01-2021, Accepted: 17-01-2021, Published: 20-01-2021
}

Abstract: The abstract must be a precise and reflection of what is in your article. Manufacturing sector is moving towards industry 4.0 and demands a high end of automation in the process. In which industrial robots play a fundamental role for automating the processes such as pick and place, material handling, palletizing, welding, painting, assembly lines and many more endless applications. Increasing demand and necessity made more research on industrial robots, machine learning and artificial intelligence. Better kinematic analysis of robots leads to reliable, high precise and fast responsive system. But there is an absence of India based robot manufacturers to fulfil the rising demand. Again, this situation leads to a market for foreign robot makers instead of local players. Lack of knowledge in robotics, unavailability of robot parts and resources are pain points for this cause. As researchers in this domain and have a goal to resolve this issue by providing open source, easily accessible industrial robot technical resources to everyone. This research work focuses the design and development of 6 Degrees of Freedom articulated robot arm with kinematic analysis particularly forward and inverse kinematics.

Keywords: Robotics, 6DoF, Articulated robot, Welding, Process automation, Robot kinematics.

\section{Introduction}

Robotics and industrial automation are important part of industry 4.0, where intervention of human is very minimal. If you consider global industrial robot market, it is raised upto USD 21.83 billion and it is projected to reach USD 66.48 billion by 2027 with CAGR of $15.1 \%$ [1] and it is shown in fig 1 .

As per research carried out by Mordor Intelligence, Asia region has highest robotics market growth rate compare to rest of the world [2] and shown in fig 2.

These market research reports clearly shows the demand and necessity of automating the different processes with help of industrial robot will make significant impact in Manufacturing Industry. India has very good infrastructure for automobile manufacturing and these manufacturing plants are automated by industrial robots in near future. But there is an absence of India based robot manufacturer to fulfil the demand.

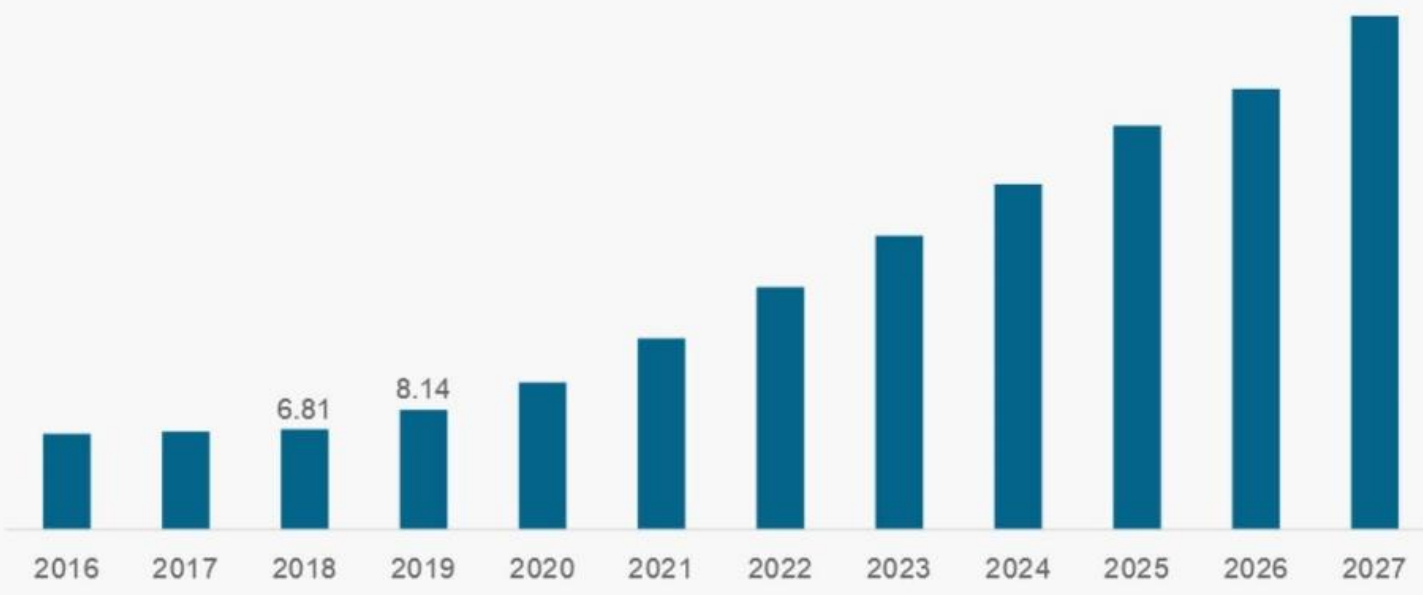

Figure 1. Asia Pacific Industrial Robots Market Size, 2016-2027 (USD Billion) 

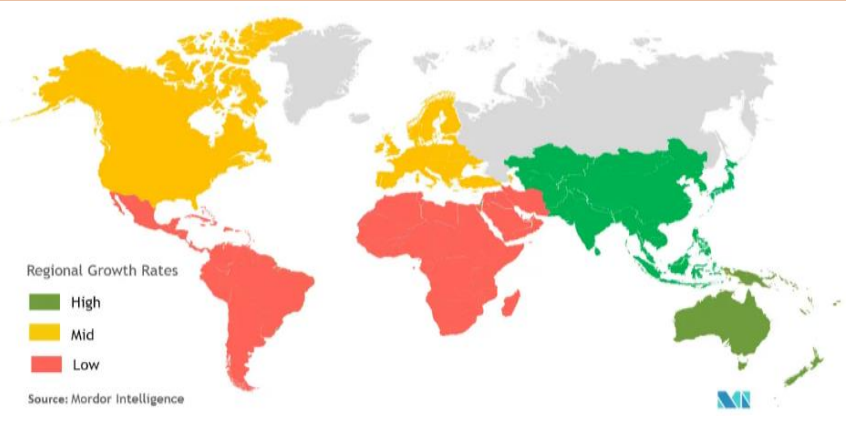

Figure 2. Robotics Market - Growth rate by region (2020-2025)

Again this situation leads to market for foreign robot makers. Lack of knowledge in robotics and unavailability of robot parts and resources are pain points for this cause. As a researcher have a have a goal to resolve this issue by providing open source, easily accessible industrial robot technical resources to everyone.

\section{Methodology}

Methodology followed in the robotic arm development is represented in Fig 3. Most of the industrial robots are articulated type configurations because it has its own advantage compared to other configurations. This articulated type construction has spherical workspace and is able to cover more volume and is suitable for many applications as mentioned earlier. Payload of the robot is selected as $2 \mathrm{~kg}$ and horizontal reach of robot is $600 \mathrm{~mm}$ in order to reduce the robot construction cost. The end effector is designed based on the application requirements.

Forward and inverse kinematics of the robot arm is derived using the $\mathrm{D}-\mathrm{H}$ parameter and Euler angle method. Orientation of the robot is defined with the help of quaternions and Slerp (Spherical linear interpolation). Different path interpolations of line, circle, spline and point to point movement are defined with parametric equations. Self-Collision and collision with the environment are monitored with the help of workspace monitoring algorithms. Robot's own zones e.g. joints and links, safe and forbidden zones are defined as cubes and capsules. The static path defined in the path planning section is represented in function of time by sampling points from the geometry along time. For trajectory generation S-Curve profile is selected for smooth motion of the robot. When compared to other profiles like Sinusoidal and Bezier curves it has shortest path time.

During the static and dynamic analysis of the robot the motor torque requirements for each joint are calculated using Lagrange and Newton methods. Communication between robot and operator is carried out by robot programming using python language.
Most of the industrial robots are actuated by AC Servo Motors but in this application stepper motors are selected to reduce the cost and are easy to control. Arduino mega 2560 is selected as robot controller. Industrial robots are equipped with strain wave reducers and cycloidal gear boxes in order to achieve higher torque demand in a small volume and very low backlash. Low cost and availability, planetary gearbox is selected. Laser tracker is used for industrial robot calibration in order to achieve repeatability prescribed in ISO 9283 [3-6]. Robot simulation is carried out in ROS (Robot Operating System) environment.

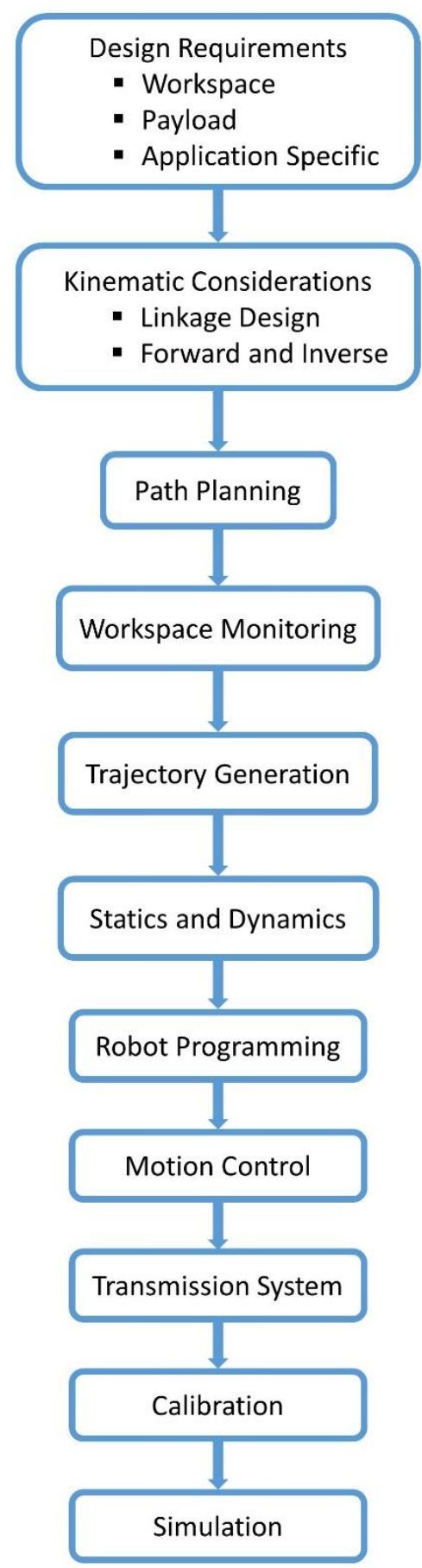

Figure 3. Methodology flow chart followed for robotic arm development 


\section{Modelling of 6 DoF Robot}

Six DOF (degrees of freedom) is selected in order to achieve any position and orientation in the given spherical volume. All joints are revolute joints with 1 degrees of freedom. 3D model of the robotic arm is developed in Solidworks CAD (Computer aided design) software and it is represented in Fig 4. Based on the selection of Motors, gearboxes, bearings, Timing belt and pulley, lead screw and nut, fasteners, robot links, robot joints, each and every parts of the robot are modelled accurately in the CAD environment.

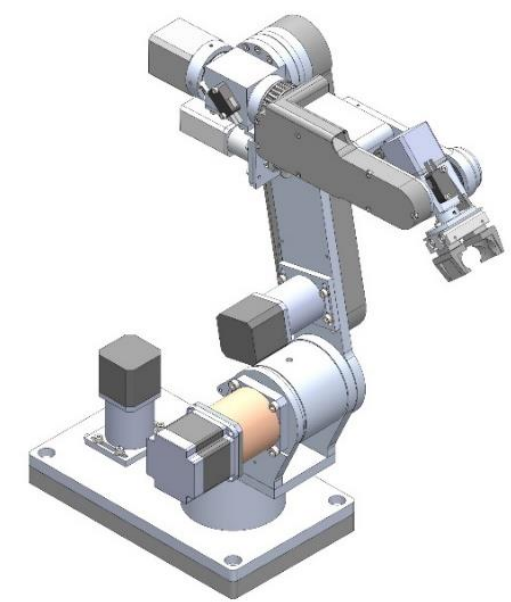

Figure 4. Computer Aided Design of 6DOF robotic arm

All six joints are actuated by stepper motor and planetary gearbox coupled system. In order to maintain the CG of the robot in the correct manner different type of Transmission techniques are followed. First, third and fourth joints are driven by timing belt and Pulley arrangement. Second and sixth joints are directly coupled to the joint axis without any second level of transmission arrangement. Fifth joint has lead screw and nut, timing belt and pulley arrangement to reduce the load on the end effector region.

Most of the robot parts are made up of aluminium 6063 and joint covers and link covers are made up of $3 D$ printed ABS plastic. Machining of the aluminium parts is carried out by Computer Numerical Controlled VMC and Turning Centers. Fused Deposition Modeling technique is followed for 3D printing process.

\section{Forward Kinematics}

In the forward kinematics process robot position and orientation are derived from the joint parameters like joint angle, length of the Prismatic joint. Denavit-Hartenberg conventional (DH parameters) method is followed to define the coordinate transformations [7]. There are four parameters to define transformation between coordinates attached to the joints between two links such as joint angle, joint offset, link length and twist angle [8].

Developed robot arm's forward kinematic representation is shown in fig 5 . The wireframe model of the robot arm is used to find out the $\mathrm{DH}$ parameter easily and the developed robot's wireframe model is represented in fig 6. Based on the $\mathrm{DH}$ convention, the four parameters for the robotic arm are listed in the table 1.

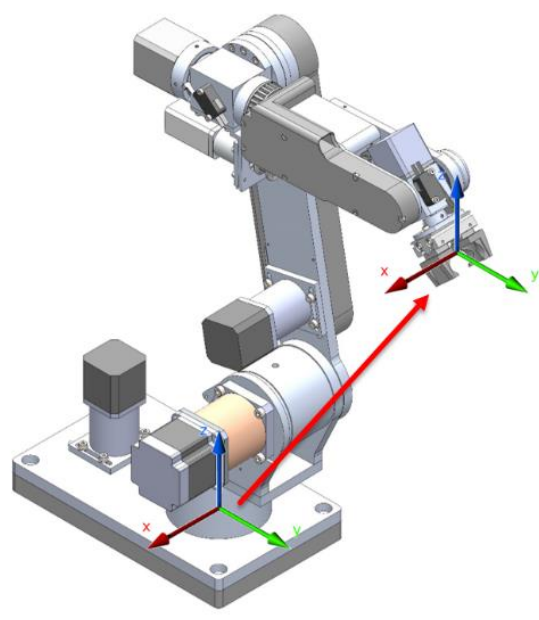

Figure 5. Forward kinematic representation

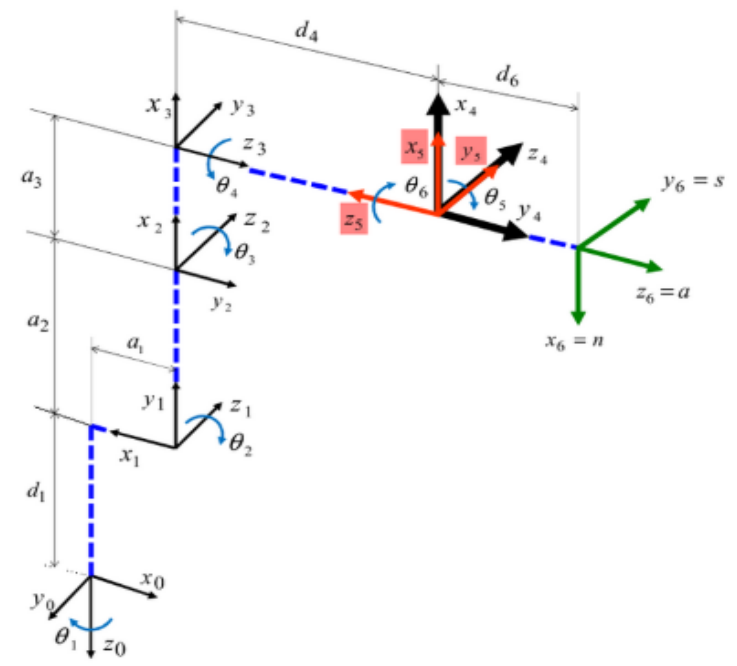

Figure 6. Forward kinematic representation

Table 1. DH Parameters of the robotic arm

\begin{tabular}{|l|l|l|l|l|}
\hline Joint & $\boldsymbol{\theta}$ & $\boldsymbol{a}$ & $\mathbf{d}$ & $\mathbf{a}$ \\
\hline $\mathbf{1}$ & 0 & -90 & 169.77 & 64.2 \\
\hline $\mathbf{2}$ & -90 & 0 & 0 & 305 \\
\hline $\mathbf{3}$ & 0 & 90 & 0 & 0 \\
\hline $\mathbf{4}$ & 0 & -90 & -222.63 & 0 \\
\hline $\mathbf{5}$ & 0 & 90 & 0 & 0 \\
\hline $\mathbf{6}$ & 180.01 & 0 & -36.25 & 0 \\
\hline
\end{tabular}


Transformation between two joints is derived by the 4X4 transformation Matrix represented in equation 1. Using an equation 2, the relation between the robot-base to the end effector position is derived in the form of $4 X 4$ transformation matrix. Euler angle of rotation is followed to find out the orientation angles using equation 3,4 and 5 .

$D H_{i-1}=\left[\begin{array}{cccc}\cos \left(\theta_{i}\right) & -\sin \left(\theta_{i}\right) \cos \left(\alpha_{i}\right) & \sin \left(\theta_{i}\right) \sin \left(\alpha_{i}\right) & a_{i} \cos \left(\theta_{i}\right) \\ \sin \left(\theta_{i}\right) & \cos \left(\theta_{i}\right) \cos \left(\alpha_{i}\right) & -\cos \left(\theta_{i}\right) \sin \left(\alpha_{i}\right) & a_{i} \sin \left(\theta_{i}\right) \\ 0 & \sin \left(\alpha_{i}\right) & \cos \left(\alpha_{i}\right) & d_{i} \\ 0 & 0 & 0 & 1\end{array}\right]$

$\mathrm{T}_{0-6}=\mathrm{T}_{0}{ }^{*} \mathrm{~T}_{1}{ }^{*} \mathrm{~T}_{2}{ }^{*} \mathrm{~T}_{3}{ }^{*} \mathrm{~T}_{4}{ }^{*} \mathrm{~T}_{5}{ }^{*} \mathrm{~T}_{6}$

$\beta=\operatorname{Atan} 2\left(-r_{31}, \sqrt{r_{11}^{2}+r_{21}^{2}}\right)$

$\alpha=\operatorname{Atan} 2\left(r_{21} / c \beta, r_{11} / c \beta\right)$

$\gamma=\operatorname{Atan} 2\left(r_{32} / c \beta, r_{33} / c \beta\right)$

\section{Inverse Kinematics}

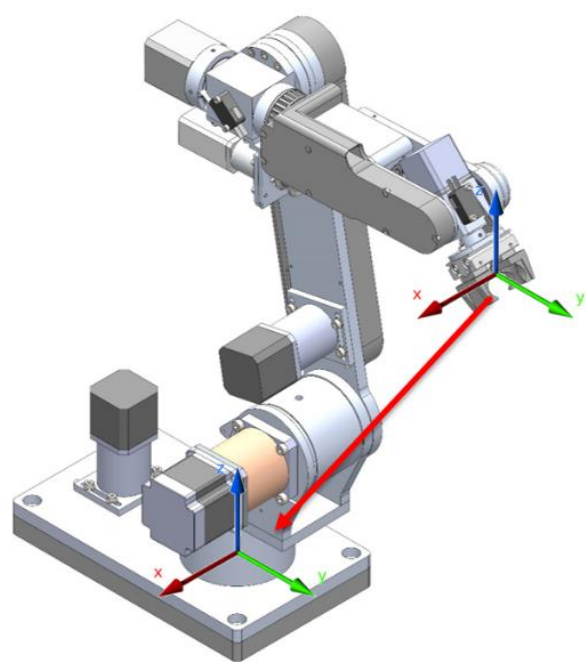

Figure 7. Inverse kinematic representation

In the Inverse kinematics, joint parameters are derived for given position and orientation of the robot i.e. Tool Centre Point (TCP) to Joint angle. Representation of inverse kinematics is shown in fig 7. First three joints result in the robot position and balance three joints causes robot orientation. Kinematic decoupling methodology is followed to find out the inverse Kinematics of the developed robot arm. The first three joint angles for the given position are derived using algebraic method. There are two different postures possible such as forward and mid. The graphical representation of the first three joints are shown in fig 8 and fig 9.

Using Euler Angle set convention shown in equation 6 , the transformation matrix is derived for given orientation. Then Joint 4, 5 and 6 parameters are calculated using an algebraic method and it is given in equations 7,8 and 9 .

$R_{Z^{\prime} X^{\prime} Z^{\prime}}(\alpha, \beta, \gamma)=\left[\begin{array}{ccc}-s \alpha c \beta s \gamma+c \alpha c \gamma & -s \alpha c \beta c \gamma-c \alpha s \gamma & s \alpha s \beta \\ c \alpha c \beta s \gamma+s \alpha c \gamma & c \alpha c \beta c \gamma-s \alpha s \gamma & -c \alpha s \beta \\ -s \beta s \gamma & s \beta c \gamma & c \beta\end{array}\right]$
$\theta_{5}=\operatorname{Atan} 2\left(r_{33}, \pm \sqrt{1-r_{33}^{2}}\right)$

$\theta_{4}=\operatorname{Atan} 2\left( \pm r_{13}, \pm r_{23}\right)$

$\theta_{6}=\operatorname{Atan} 2\left( \pm r_{31}, \pm r_{32}\right)$

There are different kinds of inverse kinematics solving methodologies are available. But this decoupling procedure will reduce singularities and also the calculation time. Based on each joint's different angle of rotation, the singularities and different possibilities of robot posture are calculated during each process.

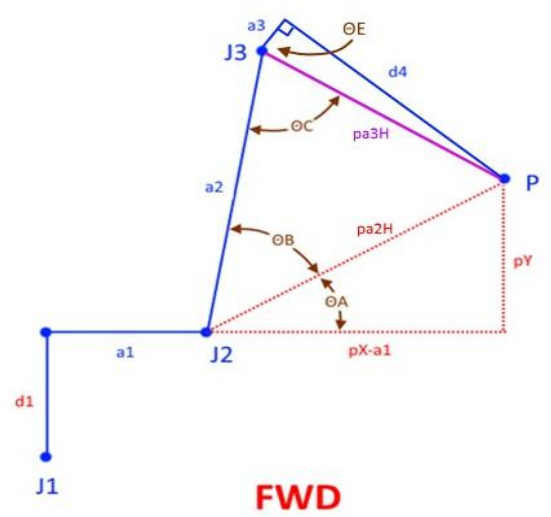

Figure 8. Forward position of robotic arm

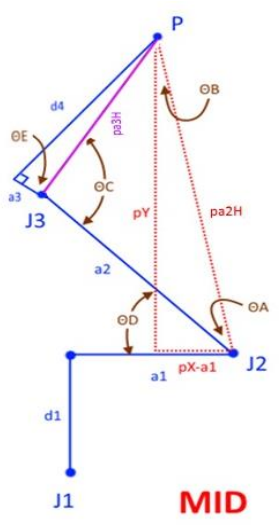

Figure 9. Mid position of robotic arm

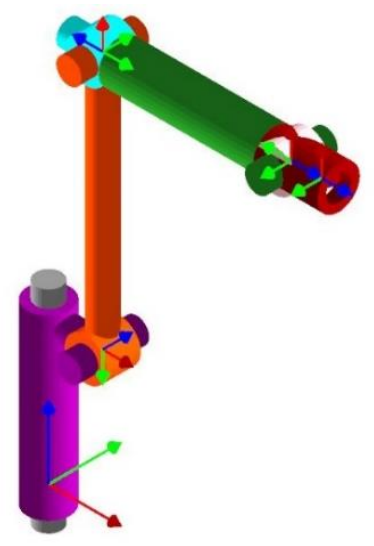

Figure 10. Robot Model in RoboAnalyzer 


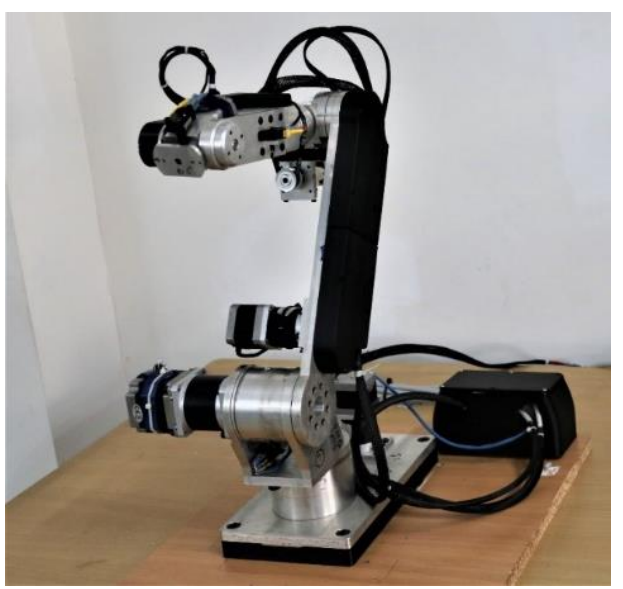

Figure 11. Developed robotic arm in real time

\section{Results and Discussion}

Forward and Inverse kinematics calculations are verified with Roboanalyser software where the developed robot is clearly modelled and all the $\mathrm{DH}$ parameters are given. RoboAnalyzer model is shown in fig 10. Homogeneous Transformation matrix of each joints and base to TCP of the robot arm are verified. The same transformations were implemented in the developed robot shown in fig 11 and the testing results of forward and inverse kinematics outputs are verified with simulation results.

\section{Conclusion}

Implementation of forward and inverse kinematics of the 6 degrees of freedom articulated robot arm is proposed by using DH parameter, Euler angle convention and kinematic decoupling to relate robot arm base to TCP. Kinematic transformations are verified with Roboanalyser software simulation. Methodology section states different types of robot parts used in industrial robots. The proposed kinematic calculations proves the robustness by simulation results and it's suitable for articulated type industrial robots.

\section{References}

[1] Industrial Robots Market by Fortune Business Insights [online] Available on Internet: https://www.fortunebusinessinsights.com/industryreports/industrial-robots-market-100360

[2] Robotics Market - Growth, Trends, COVID-19 Impact, and Forecasts (2021 - 2026) by Mordor Intelligence [online] Available on Internet: https://www.mordorintelligence.com/industryreports/robotics-market

[3] A. Nubiola, I.A. Bonev, Absolute calibration of an ABB IRB 1600 robot using a laser tracker, Robotics and Computer-Integrated Manufacturing, 29(2013) 236-245. https://doi.org/10.1016/j.rcim.2012.06.004

[4] International Organization for Standardization, Case postale $56 \mathrm{CH}-1211$ Geneva 20 Switzerland.

[5] "Manipulating industrial robots - Safety" European Standard, EN 775:1992, October1992

[6] Ivan Kuric, Vladimír Tlach, Zuzana Ságová, Miroslav Císar, Igor Gritsuk, Measurement of industrial robot pose repeatability, MATEC Web of Conferences 244, $01015 \quad$ (2018) https://doi.org/10.1051/matecconf/201824401015

[7] S.K. Saha, Introduction to Robotics, Tata McGraw Hill, 2008

[8] A.A. Hayat, R.G. Chittawadigi, A.D. Udai, S.K. Saha. (2013) Identification of Denavit-Hartenberg Parameters of an Industrial Robot. In Proceedings of Conference on Advances in Robotics (AIR '13). Association for Computing Machinery, New York, NY, USA, 1-6.

https://doi.org/10.1145/2506095.2506121

[9] J.J Craig, Introduction to Robotics - Mechanics and Control, Pearson Education Inc., 2005

\section{Acknowledgement}

The authors acknowledge the work carried out by Mr. Chris Annin in the field of Robotic and Automation. His development work is available in https://github.com/Chris-Annin

\section{Funding}

No funding was received for conducting this study.

\section{Conflict of interest}

The authors have no conflicts of interest to declare that they are relevant to the content of this article.

\section{About the License}

(C) The author(s) 2021. The text of this article is open access and licensed under a Creative Commons Attribution 4.0 International License

\section{Cite this Article}

T. Aravinthkumar, M. Suresh, B. Vinod, Kinematic Analysis of 6 DOF Articulated Robotic Arm, International Research Journal of Multidisciplinary Technovation, Vol 3, Iss 1 (2021) 1-5. DOI: https://doi.org/10.34256/irjmt2111 